\title{
Spectrum sensing for cognitive radio exploiting spectrum discontinuities detection
}

\author{
Wael Guibene ${ }^{1 *}$, Monia Turki ${ }^{2}$, Bassem Zayen ${ }^{1}$ and Aawatif Hayar ${ }^{3}$
}

\begin{abstract}
This article presents a spectrum sensing algorithm for wideband cognitive radio exploiting sensed spectrum discontinuity properties. Some work has already been investigated by wavelet approach by Giannakis et al., but in this article we investigate an algebraic framework in order to model spectrum discontinuities. The information derived at the level of these irregularities will be exploited in order to derive a spectrum sensing algorithm. The numerical simulation show satisfying results in terms of detection performance and receiver operating characteristics curves as the detector takes into account noise annihilation in its inner structure.
\end{abstract}

Keywords: cognitive radio, spectrum sensing, algebraic detection technique, low SNRs, high performances

\section{Introduction}

During the last decades, we have witnessed a great progress and an increasing need for wireless communications systems due to costumers demand of more flexible, wireless, smaller, more intelligent, and practical devices explaining markets invaded by smart-phones, personal digital assistant (PDAs), tablets and netbooks. All this need for flexibility and more "mobile" devices lead to more and more needs to afford the spectral resources that shall be able to satisfy costumers need for mobility. But, as wide as spectrum seems to be, all those needs and demands made it a scarce resource and highly misused.

Trying to face this shortage of radio resources, telecommunication regulators, and standardization organisms recommended sharing this valuable resource between the different actors in the wireless environment. The federal communications commission (FCC), for instance, defined a new policy of priorities in the wireless systems, giving some privileges to some users, called primary users (PU) and less to others, called secondary users (SU), who will use the spectrum in an opportunistic way with minimum interference to $\mathrm{PU}$ systems.

Cognitive radio (CR) as introduced by Mitola [1], is one of those possible devices that could be deployed as

\footnotetext{
* Correspondence: wael.guibene@eurecom.fr

${ }^{1}$ Mobile Communications Department, EURECOM Sophia Antipolis, France Full list of author information is available at the end of the article
}

SU equipments and systems in wireless networks. As originally defined, a CR is a self aware and "intelligent" device that can adapt itself to the Wireless environment changes. Such a device is able to detect the changes in wireless network to which it is connected and adapt its radio parameters to the new opportunities that are detected. This constant track of the environment change is called the "spectrum sensing" function of a CR device.

Thus, spectrum sensing in CR aims in finding the holes in the PU transmission which are the best opportunities to be used by the SU. Many statistical approaches already exist. The easiest to implement and the reference detector in terms of complexity is still the energy detector (ED). Nevertheless, the ED is highly sensitive to noise and does not perform well in low signal to noise ratio (SNR). Other advanced techniques based on signals modulations and exploiting some of the transmitted signals inner properties were also developed. For instance, the detector that exploits the built-in cyclic properties on a given signal is the cyclostationary features detector (CFD). The CFD do have a great robustness to noise compared to ED but its high complexity is still a consequent draw back. Some other techniques, exploiting a wavelet approach to efficient spectrum sensing of wideband channels were also developed [2].

The rest of the article is organized as following. In Section 2, we introduce the state of the art and the motivations behind our proposed approach. In Section 3 , we state the problem as a detection problem with the 
formalism related to both sensing and detection theories. The derivation of the proposed technique and some key points on its implementation are introduced in Section 4. In Section 5, we give the results and the simulation framework in which the developed technique was simulated. Finally, Section 6 summarizes about the presented work and concludes about its contributions.

\section{State of the art}

As previously stated, CR is presented [3] as a promising technology in order to handle this shortage and misuse of spectral resources. The main functions of CRs are:

- Spectrum sensing: which is an important requirement towards CR implementation and feasibility. Three main strategies do exist in order to perform spectrum sensing: transmitter detection (involving PU detection techniques), cooperative detection (involving centralized and distributed schemes) and interference based detection.

- Spectrum management: which captures the most satisfying spectrum opportunities in order to meet both PU and SU quality of service (QoS).

- Spectrum mobility: which involves the mechanisms and protocols allowing frequency hopes and dynamic spectrum use.

- Spectrum sharing: which aims at providing a fair spectrum sharing strategy in order to serve the maximum number of SUs.

The presented work fits in the context of spectrum sensing framework for CR networks (CRN) and more precisely single node detection or transmitter detection. In this context, many statistical approaches for spectrum sensing have been developed. The most performing one is the cyclostationary features detection technique $[4,5]$. The main advantage of the cyclostationarity detection is that it can distinguish between noise signal and PU transmitted data. Indeed, noise has no spectral correlation whereas the modulated signals are usually cyclostationary with non null spectral correlation due to the embedded redundancy in the transmitted signal. The CFD is thus able to distinguish between noise and PU.

The reference sensing method is the ED [4], as it is the easiest to implement. Although the ED can be implemented without any need of apriori knowledge of the PU signal, some difficulties still remain for implementation. First of all, the only PU signal that can be detected is the one having an energy above the threshold. So, the threshold selection in itself can be problematic as the threshold highly depends on the changing noise level and the interference level. Another challenging issue is that the energy detection approach cannot distinguish the PU from the other SU sharing the same channel. CFD is more robust to noise uncertainty than an ED. Furthermore, it can work with lower SNR than ED.

More recently, a detector based on the signal space dimension based on the estimation of the number of the covariance matrix independent eigenvalues has been developed [6-8]. It was presented that one can conclude on the nature of this signal based on the number of the independent eigenvectors of the observed signal covariance matrix. The Akaike information criterion (AIC) was chosen in order to sense the signal presence over the spectrum bandwidth. By analyzing the number of significant eigenvalues minimizing the AIC, one is able to conclude on the nature of the sensed sub-band. Specifically, it is shown that the number of significant eigenvalues is related to the presence or not of data in the signal.

Some other techniques, exploiting a wavelet approach to efficient spectrum sensing of wideband channels were also developed [2]. The signal spectrum over a wide frequency band is decomposed into elementary building blocks of subbands that are well characterized by local irregularities in frequency. As a powerful mathematical tool for analyzing singularities and edges, the wavelet transform is employed to detect and estimate the local spectral irregular structure, which carries important information on the frequency locations and power spectral densities of the subbands. Along this line, a couple of wideband spectrum sensing techniques are developed based on the local maxima of the wavelet transform modulus and the multi-scale wavelet products.

The proposed method was inspired from algebraic spike detection in electroencephalograms (EEGs) [9] and the recent work developed by Giannakis based on wavelet sensing [2]. Originally, the algebraic detection technique was introduced $[9,10]$ to detect spike locations in EEGs. And thus it can be used to detect signals transients. Given Gi-annakis work on wavelet approach, and its limitations in complexity and implementation, we suggest in this context of wideband channels sensing, a detector using an algebraic approach to detect and estimate the local spectral irregular structure, which carries important information on the frequency locations and power spectral densities of the subbands.

This article summarizes the work we've been conducting in spectrum sensing for CRN. A complete description of the reported work can be found in [11-15].

\section{System model}

In this section we investigate the system model considered through this article. In this system, the received signal at time $n$, denoted by $y_{n}$, can be modeled as:

$$
y_{n}=A_{n} s_{n}+e_{n}
$$


where $A_{n}$ being the transmission channel gain, $s_{n}$ is the transmit signal sent from primary user and $e_{n}$ is an additive corrupting noise.

In order to avoid interferences with the primary (licensed) system, the CR needs to sense its radio environment whenever it wants to access available spectrum resources. The goal of spectrum sensing is to decide between two conventional hypotheses modeling the spectrum occupancy:

$$
y_{n}= \begin{cases}e_{n} & \mathrm{H}_{0} \\ A_{n} s_{n}+e_{n} & \mathrm{H}_{1}\end{cases}
$$

The sensed sub-band is assumed to be a white area if it contains only a noise component, as defined in $\mathrm{H}_{0}$; while, once there exist primary user signals drowned in noise in a specific band, as defined in $\mathrm{H}_{1}$, we infer that the band is occupied. The key parameters of all spectrum sensing algorithms are the false alarm probability $P_{F}$ and the detection probability $P_{D} . P_{F}$ is the probability that the sensed sub-band is classified as a PU data while actually it contains noise, thus $P_{F}$ should be kept as small as possible. $P_{D}$ is the probability of classifying the sensed sub-band as a PU data when it is truly present, thus sensing algorithm tend to maximize $P_{D}$. To design the optimal detector on Neyman-Pearson criterion, we aim on maximizing the overall $P_{D}$ under a given overall $P_{F}$. According to those definitions, the probability of false alarm is given by:

$$
P_{F}=P\left(\mathrm{H}_{1} \mid \mathrm{H}_{0}\right)=P\left(\mathrm{PU} \text { is detected } \mid \mathrm{H}_{0}\right)
$$

that is the probability of the spectrum detector having detected a signal given the hypothesis $H_{0}$, and $P_{D}$ the probability of detection is expressed as:

$$
\begin{aligned}
P_{D} & =1-P_{M}=1-P\left(\mathrm{H}_{0} \mid \mathrm{H}_{1}\right) \\
& =1-P\left(\text { PU is not detected } \mid \mathrm{H}_{1}\right)
\end{aligned}
$$

which represents the probability of the detector having detected a signal under hypothesis $H_{1}$, where $P_{M}$ indicates the probability of missed detection.

In order to infer on the nature of the received signal, we use a decision threshold which is determined using the required probability of false alarm $P_{F}$ given by (3.3). The threshold $T h$ for a given false alarm probability is determined by solving the equation:

$$
P_{F}=P\left(y_{n} \text { is present } \mid \mathrm{H}_{0}\right)=1-F_{H_{0}}(T h)
$$

where $F_{H_{0}}$ denote the cumulative distribution function (CDF) under $\mathrm{H}_{0}$. In this article, the threshold is determined for each of the detectors via a Monte Carlo simulation.

\section{Mathematical background}

In this section some noncommutative ring theory notions are used [16]. We start by giving an overview of the mathematical background leading to the algebraic detection technique. First let's suppose that the frequency range available in the wireless network is $B \mathrm{~Hz}$; so $B$ could be expressed as $B=\left[f_{0}, f_{N}\right]$. Saying that this wireless network is cognitive, means that it supports heterogeneous wireless devices that may adopt different wireless technologies for transmissions over different bands in the frequency range. A CR at a particular place and time needs to sense the wireless environment in order to identify spectrum holes for opportunistic use. Suppose that the radio signal received by the CR occupies $N$ spectrum bands, whose frequency locations and PSD levels are to be detected and identified. These spectrum bands lie within $\left[f_{1}, f_{K}\right]$ consecutively, with their frequency boundaries located at $f_{1}<f_{2}<\cdots<f_{K}$. The $n$-th band is thus defined by: $B_{n}:\left\{f \in B_{n}: f_{n-1}<f<f_{n}, n=\right.$ $2,3, \ldots, K\}$. The PSD structure of a wideband signal is illustrated in Figure 1. The following basic assumptions are adopted:

(1) The frequency boundaries $f_{1}$ and $f_{K}=f_{1}+B$ are known to the CR. Even though the actual received signal may occupy a larger band, this CR regards $\left[f_{1}\right.$, $f_{K}$ ] as the wide band of interest and seeks white spaces only within this spectrum range.

(2) The number of bands $\mathrm{N}$ and the locations $f_{2}, \ldots$, $f_{K-1}$ are unknown to the CR. They remain unchanged within a time burst, but may vary from burst to burst in the presence of slow fading.

(3) The PSD within each band $B_{n}$ is smooth and almost flat, but exhibits discontinuities from its neighboring bands $B_{n-1}$ and $B_{n+1}$. As such, irregularities in PSD appear at and only at the edges of the $K$ bands.

(4) The corrupting noise is additive white and zero mean.

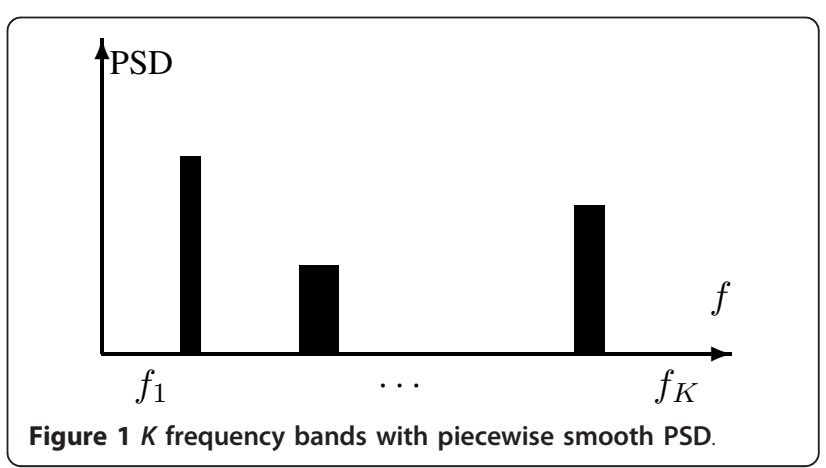


The input signal is the amplitude spectrum of the received noisy signal. We assume that its mathematical representation is a piecewise regular signal:

$$
Y(f)=\sum_{i=1}^{K} \chi_{i}\left[f_{i-1}, f_{i}\right](f) p_{i}\left(f-f_{i-1}\right)+n(f)
$$

where: $\chi_{i}\left[f_{i-1}, f_{i}\right]$ : the characteristic function of the interval $\left[f_{i-1}, f_{i}\right],\left(p_{i}\right)_{i \in[1, K]}$ : an $N$ th order polynomials series, $\left(f_{i}\right)_{i \in[1, K]}$ : the discontinuity points resulting from multiplying each $p_{i}$ by a $\chi_{i}$ and $n(f)$ : the additive corrupting noise.

Now, let $X(f)$ the clean version of the received signal given by:

$$
X(f)=\sum_{i=1}^{K} \chi_{i}\left[f_{i-1}, f_{i}\right](f) p_{i}\left(f-f_{i-1}\right)
$$

And let $b$, the frequency band, given such as in each interval $I_{b}=\left[f_{i-1}, f_{\mathrm{i}}\right]=[v, v+b], v \geq 0$ maximally one change point occurs in the interval $I_{b}$.

Now denoting $X_{v}(f)=X(f+v), f \in[0, b]$ for the restriction of the signal in the interval $I_{b}$ and redefine the change point which characterizes the distribution discontinuity relatively to $I_{b}$ say $f_{v}$ given by:

$$
y_{n}=\left\{\begin{array}{l}
f_{v}=0 \quad \text { if } X_{v} \text { is continuous } \\
0<f_{v} \leq b \text { otherwise }
\end{array}\right.
$$

Now, in order to emphasis the spectrum discontinuity behavior, we decide to use the $N$ th derivative of $X_{v}(f)$, which in the sense of distributions theory is given by:

$$
\frac{d^{N}}{d f^{N}} X_{\nu}(f)=\left[X_{v}(f)\right]^{(N)}+\sum_{k=1}^{N} \mu_{N-k} \delta\left(f-f_{v}\right)^{(k-1)}
$$

where: $\mu_{k}$ is the jump of the $k$ th order derivative at the unique assumed change point: $f_{v}$

$$
\mu_{k}=X_{v}^{(k)}\left(f_{v}^{+}\right)-X_{v}^{(k)}\left(f_{v}^{-}\right)
$$

with $\left.\mu_{k}=0\right\rfloor_{k}=1 \ldots N$ if there is no change point and $\mu_{k}$ $\neq 0\lrcorner k=1 \ldots N$ if the change point is in $I_{b}$.

$\left[X_{v}(f)\right]^{(N)}$ is the regular derivative part of the $N$ th derivative of the signal.

The spectrum sensing problem is now casted as a change point $f_{v}$ detection problem. Several estimators can be derived from the previous equations equation. For example any derivative order $N$ can be taken and depending on this order the equation is solved in the operational domain and back to frequency domain the estimator is deduced. In a matter of reducing the complexity of the frequency direct resolution, those equations are transposed to the operational domain, using the Laplace transform:

$$
\begin{aligned}
L\left(X_{v}(f)^{(N)}\right) & \left.=s^{N} \widehat{X}_{\nu}(s)-\sum_{m=0}^{N-1} s^{N-m-1} \frac{d^{m}}{d f^{m}} X_{\nu}(f)\right\rfloor f=0 \\
& =e^{-s f_{v}}\left(\mu_{N-1}+s \mu_{N-2}+\ldots+s^{N-1} \mu_{0}\right)
\end{aligned}
$$

Given the fact that the initial conditions, expressed in the previous equation, and the jumps of the derivatives of $X_{v}(f)$ are unknown parameters to the problem, in a first time we are going to annihilate the jump values $\mu_{0}, \mu_{1}, \ldots, \mu_{N-1}$ (Appendix 1) then the initial conditions (Appendix 2). After some calculations steps detailed, we finally obtain:

$$
\sum_{k=0}^{N-1}\left(\begin{array}{l}
N \\
k
\end{array}\right) \cdot f_{v}^{N-k} \cdot \mid\left(s^{N} \widehat{X}_{v}(s)\right)^{(N+k)}=0
$$

In the actual context, the noisy observation of the amplitude spectrum $Y(f)$ is taken instead of $X_{v}(f)$. As taking derivative in the operational domain is equivalent to high-pass filtering in frequency domain, which may help amplifying the noise effect. It is suggested to divide the whole previous equation by $s^{l}$ which in the frequency domain will be equivalent to an integration if $l>$ $2 N$, we thus obtain:

$$
\sum_{k=0}^{N-1}\left(\begin{array}{l}
N \\
k
\end{array}\right) \cdot f_{v}^{N-k} \cdot \frac{\left(s^{N} \widehat{X}_{v}(s)\right)^{(N+k)}}{s^{l}}=0
$$

Since here is no unknown variables anymore, the previous equation is now transformed back to the frequency domain, we obtain the polynomial to be solved on each sensed sub-band:

$$
\sum_{k=0}^{N-1}\left(\begin{array}{l}
N \\
k
\end{array}\right) \cdot f_{v}^{N-k} \cdot L^{-1}\left[\frac{\left(s^{N} \widehat{X}_{v}(s)\right)^{(N+k)}}{s^{l}}\right]=0
$$

And denoting:

$$
\varphi_{k+1}=L^{-1}\left[\frac{\left(s^{N} \widehat{X}_{v}(s)\right)^{(N+k)}}{s^{l}}\right]=\int_{0}^{+\infty} h_{k+1}(f) \cdot X(v-f) \cdot d f
$$

where: $h_{k+1}(f)= \begin{cases}\frac{\left(f^{l}(b-f)^{N+k}\right)^{(k)}}{(l-1) !} & 0<f<b \\ 0 & \text { otherwise }\end{cases}$

To summarize, we have shown that on each interval $[0, b]$, for the noise-free observation the change points are located at frequencies solving:

$$
\sum_{k=0}^{N}\left(\begin{array}{l}
N \\
k
\end{array}\right) \cdot f_{v}^{N-k} \cdot \varphi_{k+1}=0
$$

To summarize, we have shown that on each interval $[0, b]$, for the noise-free observation the change points 
are located at frequencies solving:

$$
\sum_{k=0}^{N}\left(\begin{array}{l}
N \\
k
\end{array}\right) \cdot f_{v}^{N-k} \cdot \varphi_{k+1}=0
$$

In [17], it was shown that edge detection and estimation is analyzed based on forming multiscale point-wise products of smoothed gradient estimators. This approach is intended to enhance multiscale peaks due to edges, while suppressing noise. Adopting this technique to our spectrum sensing problem and restricting to dyadic scales, we construct the multiscale product of $N$ +1 filters (corresponding to continuous wavelet transform in [17]), given by:

$$
D f=\left\|\prod_{k=0}^{N} \varphi_{k+1}\left(f_{v}\right)\right\|
$$

\subsection{Implementation issues}

The proposed algorithm is implemented as a filter bank which is composed of $N$ filters mounted in a parallel way. The impulse response of each filter is:

$$
h_{k+1}(f)= \begin{cases}\frac{\left(f^{l}(b-f)^{N+k}\right)^{(k)}}{(l-1) !} & 0<f<b \\ 0 & \text { otherwise }\end{cases}
$$

where $k \in[0 \ldots N-1]$ and $l$ is chosen such as $l>2 \times$ $N$. The proposed expression of $\left.h_{k+1}\right\rfloor_{k \in[0 . . N-1]}$ was determined by modeling the spectrum by a piecewise regular signal in frequency domain and casting the problem of spectrum sensing as a change point detection in the primary user transmission. Finally, in each stage of the filter bank, we compute the following equation:

$$
\varphi_{k+1}(f)=\int_{0}^{+\infty} h_{k+1}(v) \cdot X(f-v) \cdot d v
$$

Then, we process by detecting spectrum discontinuities and to find the intervals of interest.

\subsection{Algorithm discrete implementation}

The proposed algorithm in its discrete implementation is a filter bank composed of $N$ filters mounted in a parallel way. The impulse response of each filter is:

$$
h_{k+1, n}= \begin{cases}\frac{\left(n^{l}(b-n)^{N+k}\right)^{(k)}}{(l-1) !} & 0<n<b \\ 0 & \text { otherwise }\end{cases}
$$

where $k \in[0 \ldots N-1]$ and $l$ is chosen such as $l>2 \times$ $N$. The proposed expression of $\left.h_{k+1, n}\right\rfloor_{k \in[0 \ldots N-1]}$ was determined by modeling the spectrum by a piecewise regular signal in frequency domain and casting the problem of spectrum sensing as a change point detection in the primary user transmission. Finally, in each detected interval $\left[n_{v_{i}}, n_{v_{i+1}}\right]$, we compute the following equation:

$$
\varphi_{k+1}=\sum_{m=n_{v_{i}}}^{n_{v_{i+1}}} W_{m} h_{k+1, m} X_{m}
$$

where $W_{m}$ are the weights for numeric integration defined by:

$$
\begin{aligned}
W_{0} & =W_{M}=0.5 \\
W_{m} & =1 \quad \text { otherwise }
\end{aligned}
$$

In order to infer whether the primary user is present in the detected intervals, a decision function is computed as following:

$$
D f=\left\|\prod_{k=0}^{N} \varphi_{k+1}\left(n_{v}\right)\right\|
$$

\section{Performance evaluation}

\subsection{Performance metrics}

Receiver operating characteristic (ROC) is a curve that shows comparison of the probability of correct detection $\left(P_{D}\right)$ versus the probability of false alarm $\left(P_{F A}\right)$. Such curve is standard way for verification of a detection algorithms. AD technique has been compared to the ED considered as a reference technique. Each point is constructed by averaging results from 1,000 simulations and the change of detection probability has been achieved by changing the algorithms threshold level. An estimate of $P_{D}, \hat{P}_{D}$ can be expressed as:

$$
\hat{P}_{D}=\frac{\sum_{i=1}^{1000} N_{c d}^{(i)}}{\sum_{i=1}^{1000} N_{a}^{(i)}}
$$

where $N_{c d}$ is the number of correct detections per iteration and $N_{a}$ is number of generated change points per iteration (it's the same in every iteration).

Estimation of $P_{F A}, \hat{P}_{F A}$ is more complex since $N_{d}$, total number of detected change points per iteration, is not a constant. Therefore $\hat{P}_{F A}$ is calculated as a sum of fake detection probabilities for each different number of total detections, multiplied with the probability that such number of total detection occurs (weight factor in conditional probability):

$$
\hat{P}_{F A}=\sum_{k=0}^{n} \hat{P}_{F A \mid k} P\left(N_{d}=k\right)
$$


where: $\hat{P}_{F A \mid k}$ is defines as:

$$
\hat{P}_{F A \mid k}=\left\{\begin{array}{c}
\frac{\overline{N_{F A \mid k}}}{k} k \in N * \\
0 \quad \text { if } k=0
\end{array}\right.
$$

where $\overline{N_{F A \mid k}}$ is the average number of falsely detected change points given that the number of detected ones is $k$ with $n$ different realizations.

\subsection{Simulations results}

In this section, we use the ED as a reference technique, since it is the most common method for spectrum sensing because of its non-coherency and low complexity. The ED measures the received energy during a finite time interval and compares it to a predetermined threshold. That is, the test statistic of the ED is:

$$
\sum_{n=1}^{M}\left\|y_{n}\right\|^{2}
$$

where $M$ is the number of samples of the received signal $x_{n}$. Traditional ED can be simply implemented as a spectrum analyzer. A threshold used for PU detection is highly susceptible to unknown or changing noise levels. Even if the threshold would be set adaptively, presence of any in-band interference would confuse the ED.

Since the complexity of sensing algorithms is a major concern in implementation. As ED is well known for its simplicity, the comparison is made with reference to it. Denoting $M$ the number of samples of the received signal $y_{n}$ and $N$ is the model order of the AD, we show that the AD complexity is $N M$ and the ED complexity is $M$. From these results, we clearly see that the proposed sensing algorithm has a comparable complexity level as the ED. Table 1 summarizes the complexity of the two techniques.

For simulation results, the choice of the DVB-T PU system is justified by the fact that most of the PU systems utilize the OFDM modulation format [18]. The considered model is an additive white Gaussian noise (AWGN) channel. The simulation scenarios are generated by using different combinations of parameters given in Table 2.

Figure 2 shows the detected change points by the algebraic technique where: the blue signal is the simulated OFDM signal and the green stars are the detected change points.

Figure 3 reports the comparison in terms of Probability of Detection versus SNR between the ED $(E D)$ and the three first algebraic detectors: $\left(A D_{1}\right)\left(A D_{2}\right)$ and $\left(A D_{3}\right)$, for $P_{F}=0.05$ and SNR ranging in -40 to $0 \mathrm{dBs}$. The threshold level for each detector is computed with function of the probability of false alarm $P_{F}$ with respect
Table 1 Complexity comparison of the different sensing techniques

\begin{tabular}{ll}
\hline Sensing technique & Complexity \\
\hline Energy detector & $\mathrm{M}$ \\
Algebraic detector & $\mathrm{NM}$ \\
\hline
\end{tabular}

to (3.5). This figure clearly shows that the proposed sensing algorithm is quite robust to noise. These curves show also that the detection rate goes higher as the polynomial order gets higher. This result is to be expected as the higher the polynomial order is, the more accurate the approximation a polynomial is. Nevertheless, it is to be noticed that this gain in precision is implies a higher complexity in the algorithms implementation.

In Figure 4, we plot the ROC curve at an SNR $=-15$ $\mathrm{dB}$. We clearly see that for the proposed technique, the higher the order, the more performing the detector gets.

\section{Conclusion}

In this article, we presented a new standpoint for spectrum sensing emerging in detection theory, deriving from differential algebra, noncommutative ring theory, and operational calculus. The proposed algebraic based algorithm for spectrum sensing by change point detections in order to emphasizes "spike-like" parts of the given noisy amplitude spectrum. Simulations results showed that the proposed approach is very efficient to detect the occupied sub-bands in the the primary user transmissions. We have shown how very simple sensing algorithm with good robustness to noise can be devised within the framework of such unusual mathematical chapters in signal processing. A probabilistic interpretation, in the sense of ROC curve, probability of detection and probability of false alarm, is shown to be attached to the presented approach. It has allowed us to give a first step towards a more complete analysis of the proposed sensing algorithms.

\section{Appendix 1. Annihilating jumps in the derivatives} In a matter of reducing the complexity of the frequency direct resolution, the involved equations are transposed to the operational domain, using the Laplace transform.

Table 2 The transmitted DVB-T primary user signal parameters

\begin{tabular}{ll}
\hline Bandwidth & $8 \mathrm{MHz}$ \\
Mode & $2 \mathrm{~K}$ \\
Guard interval & $1 / 4$ \\
Frequency-flat & Single path \\
Sensing time & $1.25 \mathrm{~ms}$ \\
Location variability & $10 \mathrm{~dB}$ \\
\hline
\end{tabular}




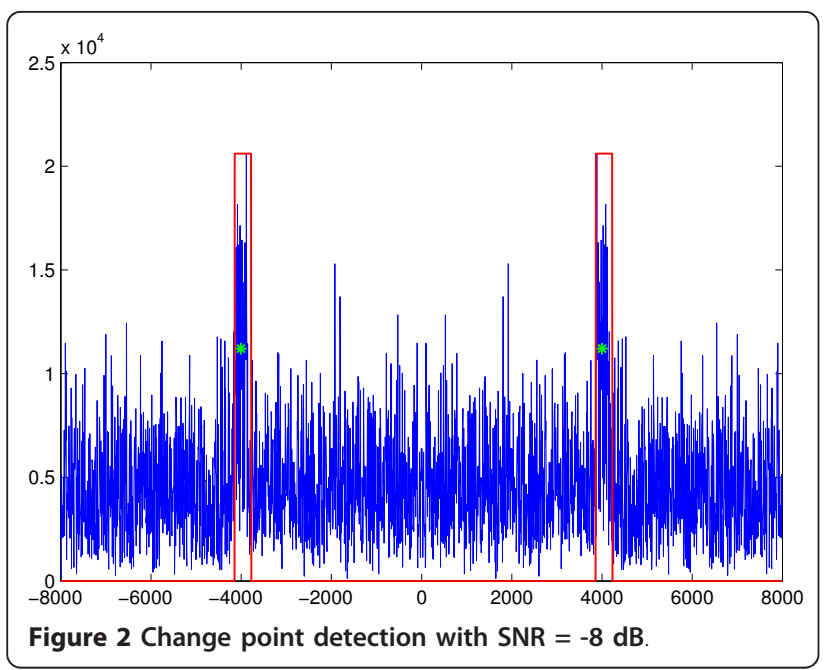

The equation in the operational domain is given by:

$$
\begin{aligned}
& \left.L\left(X_{\nu}(f)^{(N)}\right)=s^{N} \widehat{X}_{\nu}(s)-\sum_{m=0}^{N-1} s^{N-m-1} \frac{d^{m}}{d f^{m}} X_{\nu}(f)\right\rfloor_{f=0} \\
& =e^{-s f_{\nu}}\left(\mu_{N-1}+s \mu_{N-2}+s \mu_{N-3}+\ldots+s^{N-1} \mu_{0}\right)
\end{aligned}
$$

Given the fact the initial conditions and the jump of the derivatives of $X_{v}(f)$ are unknown parameters to the problem, in a first time we are going to annihilate the jump values $\mu_{0}, \mu_{1}, \ldots, \mu_{N-1}$ then the initial conditions. In order to make further calculations easier and shorter to write, let:

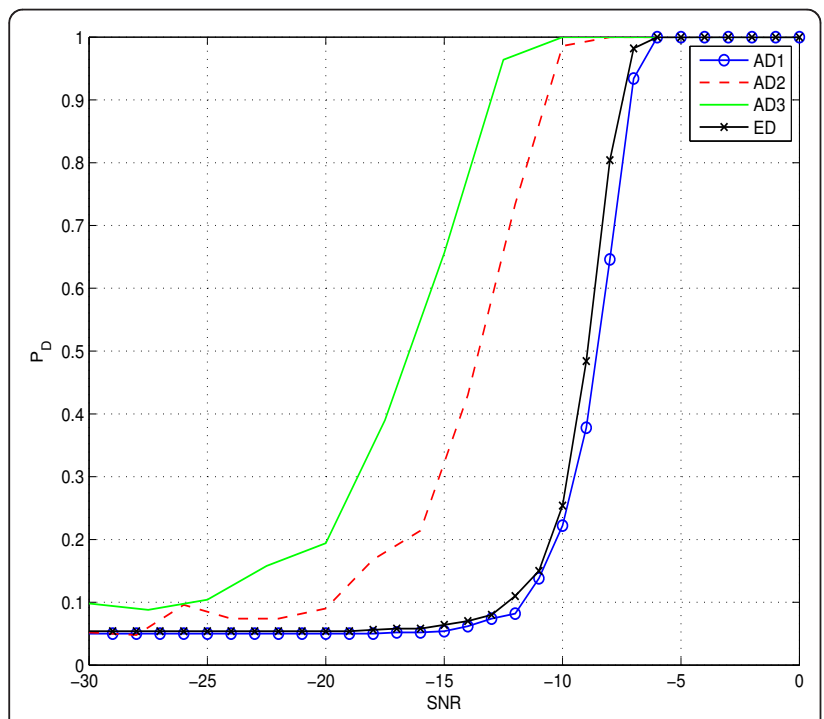

Figure 3 Probability of detection vs. SNR for the simulated detectors with $P_{F}=0.05$.

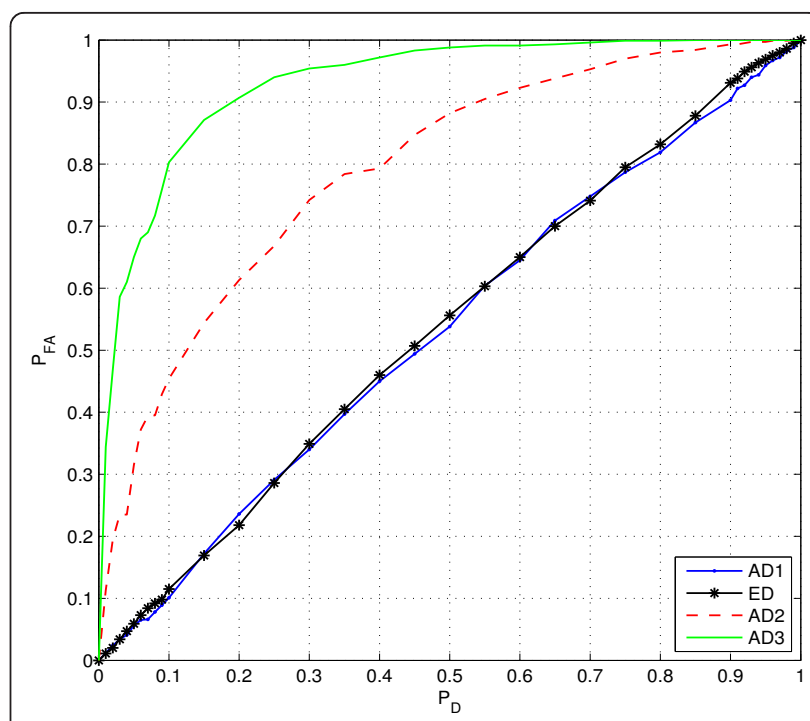

Figure 4 ROC curves at SNR $=-15 \mathrm{~dB}$

$\left.u(s)=s^{N} \widehat{X}_{v}(s)-\sum_{m=0}^{N-1} s^{N-m-1} \frac{d^{m}}{d f^{m}} X_{\nu}(f)\right\rfloor_{f=0}$, then the Equation (1.1) in Appendix 1 becomes:

$$
e^{s f_{v}} u(s)=\mu_{N-1}+s \mu_{N-2}+s^{2} \mu_{N-3}+\ldots+s^{N-1} \mu_{0}
$$

Now, a simple $N$ times derivation of the previous equation with respect to $s$ cancels the jumps $\mu_{0}, \mu_{1}, \ldots$, $\mu_{N-1}$ of the derivatives and we thus obtain:

$$
\frac{d^{N}}{d s^{N}}\left(e^{s f_{v}}(s)\right)=0
$$

Now, given the fact that both functions:

$$
\begin{aligned}
& {\left[s \mapsto e^{s f_{v}}\right]} \\
& \left.\left[s \mapsto u(s)=s^{N} X_{v}(s)-\sum_{m=0}^{N-1} s^{N-m-1} \frac{d^{m}}{d f^{m}} X_{v}(f)\right\rfloor_{f=0}\right]
\end{aligned}
$$

are $N$-times differentiable functions, using the Leibniz Theorem for generalized $N^{\text {th }}$ derivative, we obtain:

$$
\left(e^{s f_{v}} u(s)\right)^{(N)}=\sum_{k=0}^{N}\left(\begin{array}{l}
N \\
k
\end{array}\right) \cdot\left(e^{s f_{v}}\right)^{(N-k)} \cdot(u(s))^{(k)}
$$

where, $\left(\begin{array}{l}N \\ k\end{array}\right)=\frac{N !}{k !(N-k) !}::$ denotes the binomial coefficient.

That's to say:

$$
\sum_{k=0}^{N}\left(\begin{array}{l}
N \\
k
\end{array}\right) \cdot e^{s f_{v}} \cdot f_{v}^{N-k} \cdot(u(s))^{(k)}=0
$$

Now, given the fact that the initial conditions in: 


$$
\left.u(s)=s^{N} \widehat{X}_{v}(s)-\sum_{m=0}^{N-1} s^{N-m-1} \frac{d^{m}}{d f^{m}} X_{v}(f)\right\rfloor_{f=0}
$$

unknown parameters, we make $\mathrm{N}$-times derivatives of the previous equation equation to annihilate them, we thus obtain:

$$
\sum_{k=0}^{N}\left(\begin{array}{l}
N \\
k
\end{array}\right) \cdot e^{s f_{v}} \cdot f_{v}^{N-k} \cdot(u(s))^{(N+k)}=0
$$

Now, given that:

$$
\left.u(s)=s^{N} \widehat{X}_{v}(s)-\sum_{m=0}^{N-1} s^{N-m-1} \frac{d^{m}}{d f^{m}} X_{v}(f)\right\rfloor_{f=0} \text {, after } N-
$$

times derivatives only $\left(s^{N} \widehat{X}_{v}(s)\right)^{(N)}$ remains, so :

$$
\sum_{k=0}^{N}\left(\begin{array}{l}
N \\
k
\end{array}\right) \cdot e^{s f_{v}} \cdot f_{v}^{N-k} \cdot\left(s^{N} \widehat{X}_{v}(s)\right)^{(N+k)}=0
$$

\section{Appendix 2. Annihilating initial conditions}

Since there is no unknown variables anymore, the equations are now transformed back to the frequency domain using the inverse Laplace transform, we obtain the polynomial to be solved on each sensed sub-band:

$$
\sum_{k=0}^{N}\left(\begin{array}{l}
N \\
k
\end{array}\right) \cdot e^{s f_{v}} \cdot f_{v}^{N-k} \cdot L^{-1}\left[\frac{\left(s^{N} \hat{X}_{v}(s)\right)^{(N+k)}}{s^{l}}\right]=0
$$

In a matter of clarity, the equation 18 is taken back to frequency domain for the three arguments separately:

$$
L^{-1}\left[\frac{\left(s^{N} \widehat{X}_{\nu}(s)\right)^{(N+k)}}{s^{l}}\right]=\frac{1}{(1-1) !} \int_{0}^{b}(b-f)^{(l-1)} f^{N+k} X_{v}^{(N)}(f) d f
$$

Denoting the substitution $\lambda$, so that $\lambda b=f$, leads to integration borders: $\left\{\begin{array}{l}f=b \Rightarrow \lambda=1 \\ f=0 \Rightarrow \lambda=0\end{array}\right.$ and the integration becomes:

$$
\begin{aligned}
& L^{-1}\left[\frac{\left(s^{N} \widehat{X}_{v}(s)\right)^{(N+k)}}{s^{l}}\right]=\frac{1}{(l-1) !} \int_{0}^{b}(b-\lambda b)^{l-1} \lambda^{N+k} X_{v}^{(N)}(\lambda) \cdot b \cdot d \lambda \\
& L^{-1}\left[\frac{\left(s^{N} \widehat{X}_{v}(s)\right)^{(N+k)}}{s^{l}}\right]=\frac{b^{l+N+k}}{(l-1) !} \int_{0}^{1}(1-\lambda)^{l-1} \lambda^{N+k} X_{v}^{(N)}(\lambda) \cdot d \lambda
\end{aligned}
$$

In order to avoid $X_{v}^{(N)}(\lambda)$ which corresponds to a high-pass filtering, integration by parts is applied ( $N$ 1)-times with the formula:

$$
\int_{a}^{b} u \prime v=[u v]_{a}^{b}-\int_{a}^{b} u v \prime
$$

where each time: $u \prime(\lambda)=X_{v}^{(N)}(\lambda), X_{v}^{(N)}(\lambda), \ldots, X_{\nu}^{(2)}(\lambda), X_{v}^{1}(\lambda), \quad$ which gives:

$$
L^{-1}\left[\frac{\left(s^{N} \widehat{X}_{\nu}(s)\right)^{(N+k)}}{s^{l}}\right]=\frac{b^{l+N+k}}{(l-1) !} \int_{0}^{1}\left((1-\lambda)^{l-1} \lambda^{N+k}\right)^{(N)} X_{\nu}(\lambda) \cdot d \lambda
$$

Now back to the original notations, we obtain:

$$
L^{-1}\left[\frac{\left(s^{N} \widehat{X}_{\nu}(s)\right)^{(N+k)}}{s^{l}}\right]=\frac{1}{(1-1) !} \int_{0}^{b}\left((b-f)^{l-1} f^{N+k}\right)^{(N)} X_{\nu}(f) \cdot d f
$$

And as stated previously, $X_{v}(f)=X(f+v), f_{\varepsilon}[0, b]$, we thus obtain:

$$
L^{-1}\left[\frac{\left(s^{N} \widehat{X}_{\nu}(s)\right)^{(N+k)}}{s^{l}}\right]=\frac{1}{(1-1) !} \int_{0}^{b}\left((b-f)^{l-1} f^{N+k}\right)^{(N)} X(f+v) \cdot d f
$$

Now, in order to emphasize the convolution form, let's denote: $f \leftarrow b-f$ :

$$
L^{-1}\left[\frac{\left(s^{N} \widehat{X}_{\nu}(s)\right)^{(N+k)}}{s^{l}}\right]=\frac{1}{(1-1) !} \int_{0}^{b}\left(f^{l-1}(b-f)^{N+k}\right)^{(N)} X(v+b-f) \cdot d f
$$

And in order to simplify the expression let $v \leftarrow v+b$, we get the following expression:

$$
L^{-1}\left[\frac{\left(s^{N} \widehat{X}_{v}(s)\right)^{(N+k)}}{s^{l}}\right]=\int_{0}^{b} \frac{\left(f^{l-1}(b-f)^{N+k}\right)^{(N)}}{(l-1) !} X(v-f) \cdot d f
$$

Now, denoting:

$$
\begin{gathered}
\varphi_{k+1}=L^{-1}\left[\frac{\left(s^{N} \widehat{X}_{v}(s)\right)^{(N+k)}}{s^{l}}\right]=\int_{0}^{+\infty} h_{k+1}(f) \cdot X(v-f(2 d) \\
\text { where: } h_{k+1}(f)= \begin{cases}\frac{\left(f^{l-1}(b-f)^{N+k}\right)^{(N)}}{(l-1) !}, & 0<f<b \\
0, & \text { otherwise }\end{cases}
\end{gathered}
$$

To summarize, we have shown that on each interval $[0, b]$, for the noise-free observation the change points are located at frequencies solving:

$$
\sum_{k=0}^{N}\left(\begin{array}{l}
N \\
k
\end{array}\right) \cdot e^{s f_{v}} \cdot f_{v}^{N-k} \cdot \varphi_{k+1}=0
$$

And the estimator is deduced by assuming as input the real amplitude spectrum $Y(f)$ instead of $X(f)$.

\section{Acknowledgements}

The research work was carried out at EURECOM's Mobile Communications leading to these results has received funding from the European Community's Seventh Framework Programme (FP7/2007-2013) under grant agreement SACRA n॰249060. 


\section{Author details}

${ }^{1}$ Mobile Communications Department, EURECOM Sophia Antipolis, France ${ }^{2}$ Unité Siguax et Systèmes Ecole Nationale d'Ingénieurs de Tunis, BP37, Le Belvedere-1002, Tunis, Tunisia ${ }^{3}$ GREENTIC, Université Hassan II, Casablanca, Morocco

\section{Competing interests}

The authors declare that they have no competing interests.

Received: 6 July 2011 Accepted: 9 January 2012

Published: 9 January 2012

\section{References}

1. J Mitola, Cognitive radio for flexible mobile multimedia communications, in IEEE International Workshop on Mobile Multimedia Communications, (San Diego, CA, Nov. 1999), pp. 3-10

2. Z Tian, GB Giannakis, A wavelet approach to wideband spectrum sensing for cognitive radios, in IEEE 1st International Conference on Cognitive Radio Oriented Wireless Networks and Communications, CROWNCOM, (Mykonos, Greece, June 2006), pp. 1-5

3. IF Akyildiz, W-Y Lee, MC Vuran, S Mohanty, Next generation/dynamic spectrum access/cognitive radio wireless networks: a survey. Comput. Netw. J 50, 2027-2159 (2006)

4. T Yncek, H Arslan, A srvey of spectrum sensing algorithms for cognitive radio applications. IEEE Commun. Surv. Tutor. 11, 116-130 (2007)

5. S Xu, Z Zhao, J Shang, Spectrum sensing based on cyclostationarity, in Workshop on Power Electronics and Intelligent Transportation System, PEITS '08, (Guangzhou, China, Aug 2008), pp. 171-174

6. B Zayen, A Hayar, K Kansanen, Blind spectrum sensing for cognitive radio based on signal space dimension estimation, in IEEE International Conference on Communications, (Paris, France, Nov 2009), pp. 1-5

7. B Zayen, A Hayar, D Nussbaum, Blind spectrum sensing for cognitive radio based on model selection, in 3rd International Conference on Cognitive Radio Oriented Wireless Networks and Communications, CrownCom 2008, Singapore, pp. 1-5 (15-17 May 2008)

8. B Zayen, W Guibene, A Hayar, Performance comparison for low complexity blind sensing techniques in cognitive radio systems, in 2nd International Workshop on Cognitive Information Processing, CIP'10, (Elba Island, Tuscany, Italy, 14-16 June 2010), pp. 328-332

9. Z Tiganj, An algebraic delay estimation method for neuronal spike detection, (Master thesis, Zagreb University, Croatia, 2008)

10. M Mboup, C Join, M Fliss, An on-line change-point detection method. in 16th Mediterranean Conference on Control and Automation 1290-1295 (2527 June 2008)

11. W Guibene, A Hayar, Joint time-frequency spectrum sensing for cognitive radio, in 3rd International Workshop on Cognitive Radio and Advanced Spectrum Management, CogART 2010, (Rome, Italy, 7-10 Nov 2010), pp. 1-4

12. H Moussavinik, W Guibene, A Hayar, Centralized collaborative compressed sensing of wideband spectrum for cognitive radios, in International Conference on Ultra Modern Telecommunications, ICUMT 2010, (Moscow, Russia, 18-20 Oct 2010), pp. 246-252

13. B Zayen, W Guibene, A Hayar, Performance comparison for low complexity blind sensing techniques in cognitive radio systems, in 2nd International Workshop on Cognitive Information Processing CIP'10, (Elba Island, Tuscany, Italy, 14-16 June 2010), pp. 328-332

14. W Guibene, A Hayar, M Turki, Distribution discontinuities detection using algebraic technique for spectrum sensing in cognitive radio networks, in 5th International Conference on Cognitive Radio Oriented Wireless Networks and Communications, CrownCom 2010, (Cannes, France, 9-11 June 2010), pp. 1-5

15. W Guibène, H Moussavinik, A Hayar, Combined compressive sampling and distribution discontinuities detection approach to wideband spectrum sensing for cognitive radios, in International Conference on Ultra Modern Telecommunications, ICUMT 2011, (Budapest, Hungary, 5-7 Oct 2011)

16. P Moin, Fundamentals of Engineering Numerical Analysis. Chapter 1: Interpolation (Cambridge University Press, Cambridge, 2010), pp. 1-8. ISBN10:0521805260

17. Z Tian, GB Giannakis, A wavelet approach to wideband spectrum sensing for cognitive radios, in 1st International Conference on Cognitive Radio Oriented Wireless Networks and Communications, 2006, (Greece, 2006), pp. $1-5$
18. MA Ingram, G Acosta, OFDM Simulation Using Matlab, Georgia Institute of Technology. (2000)

doi:10.1186/1687-1499-2012-4

Cite this article as: Guibene et al:: Spectrum sensing for cognitive radio exploiting spectrum discontinuities detection. EURASIP Journal on Wireless Communications and Networking 2012 2012:4.

\section{Submit your manuscript to a SpringerOpen ${ }^{\mathcal{O}}$ journal and benefit from:}

- Convenient online submission

- Rigorous peer review

- Immediate publication on acceptance

- Open access: articles freely available online

- High visibility within the field

- Retaining the copyright to your article

Submit your next manuscript at $\boldsymbol{~ s p r i n g e r o p e n . c o m ~}$ 